\title{
ETL Workflow Analysis and Verification Using Backwards Constraint Propagation
}

\author{
Jie $\mathrm{Liu}^{1,2}$, Senlin Liang ${ }^{3}$, Dan $\mathrm{Ye}^{2}$, Jun $\mathrm{Wei}^{2}$, and Tao Huang ${ }^{2}$ \\ 1 University of Science and Technology of China, Anhui Hefei, China \\ 2 Institute of Software, Chinese Academy of Sciences, Beijing, China \\ $\{1 j i e, y e d a n, w j$, tao $\}$ @otcaix.iscas.ac.cn \\ 3 Department of Computer Science \\ State University of New York at Stony Brook \\ Stony Brook, NY 11794, USA \\ sliang@cs.sunysb.edu
}

\begin{abstract}
One major contribution of data warehouses is to support better decision making by facilitating data analysis, and therefore data quality is of primary importance. ETL is the process that extracts, transforms, and ultimately loads data into target warehouses. Although ETL workflows can be designed by ETL tools, data exceptions are largely left to human analysis and handled inadequately. Early detection of exceptions helps to improve the stability and efficiency of ETL workflows. To achieve this goal, a novel approach, Backwards Constraint Propagation (BCP), is proposed that automatically analyzes ETL workflows and verifies the target-end restrictions at their earliest points. BCP builds an ETL graph out of a given ETL workflow, encodes the target-end restrictions as integrity constraints, and propagates them backwards from target to sources through the ETL graph by applying constraint projection rules. It is showed that BCP supports most relational algebra operators and data transformation functions.
\end{abstract}

Keywords: ETL, Workflow Analysis, Data Quality, Data Warehouse, Constraint Propagation.

\section{Introduction}

ETL (Extract, Transform, and Load) is the important process to build data warehouses [1, and it involves identifying relevant information at data sources, extracting the relevant data, transforming it to fit business needs and ultimately loading the data into the target data warehouse. Initially, ETL processes were hard-coded, and thus difficult and expensive to maintain. Nowadays most database and data integration systems vendors offer powerful ETL tools, most of which can be classified into two categories. One provides independent engines to run ETL tasks, and its representatives are PowerCenter 1 from Informatica,

${ }^{1}$ http://www.informatica.com/products_services/powercenter/Pages/index.aspx

P. van Eck, J. Gordijn, and R. Wieringa (Eds.): CAiSE 2009, LNCS 5565, pp. 455 469, 2009.

(C) Springer-Verlag Berlin Heidelberg 2009 


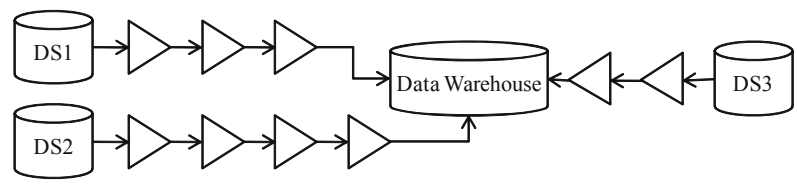

Fig. 1. An Example of ETL Workflows

Data Stage 2 from IBM, and SSIS3 from Microsoft. The other category is also named ELT (Extract, Load, Transform), which delegates the task of running ETL workflows to DBMS. Its representative is Oracle Data Integrator 4 .

An example ETL workflow is presented in Figure1, which integrates data from several data sources (DS1, DS2 and DS3) into target data warehouse by applying series of transformations (triangles). ETL workflows are designed by ETL tools manually, using schema mappings between sources and target. Due to the diversity of source schema and complexity of ETL workflows, some dirty data may cause exceptions when going through workflows or being loaded into the target.

There have been many researches in exceptional data detection and data cleaning, and most vendors provide data quality control suites together with their ETL tools. They mainly employ the following methods: data analysis, data sampling, data quality monitoring and target-end integrity checking.

Data Analysis analyzes real data instances to obtain data characteristics and value patterns, which help detect data outliers and build schema mappings [2]. There are two approaches to data analysis: data profiling and data mining. Data profiling derives the information of each attribute (e.g. type, uniqueness, null values), and data mining discovers data patterns (e.g. relationships among several attributes).

Data sampling applies ETL workflows on a sample data set to find exceptional data and improves the design of ETL workflows. Data sampling is commonly used in practice, and its performance heavily depends on the quality of the sampling data set.

Data quality monitoring imposes quality control by defining data quality rules over ETL workflows. The system decides whether to abort the ETL workflows or to perform data cleaning on detecting the violation of these rules. However, these rules have to be manually defined, and most of time it is infeasible to define rules to capture all possible exceptions in real applications.

Target-end integrity checking filters out exceptional data, which violates the target-end integrity constraints, when loading into the target warehouse and keeps a record of them for later processing.

Only target-end integrity checking can guarantee the satisfiability of all the constraints at the target. However, all involved data (exceptional or not) are

\footnotetext{
${ }^{2}$ http://www-01.ibm.com/software/data/infosphere/datastage

${ }^{3}$ http://code.msdn.microsoft.com/SSIS/Wiki/View.aspx

${ }^{4}$ http://www.oracle.com/technology/products/oracle-data-integrator/index.html
} 
transferred to the target, which incurs much extra cost. Moreover, data analysis and data quality monitoring require manual analysis of ETL processes and manual encoding of mappings between the target and sources, which are expensive, error-prone and time consuming. Thus we want an approach that can automatically: 1) analyze ETL workflows and the target-end restrictions, and verify these restrictions at their earliest possible points; 2) on the violation of these restrictions, call predefined handlers according to user specified exception handling policies.

Most restrictions at the target warehouse can be expressed by a set of integrity constraints, which are also referred as data quality rules. In order for the data in the ETL workflow to satisfy the integrity constraints at the target, it should have certain properties when it moves through the ETL workflow. These properties can be derived from the target's integrity constraints.

In this paper, we proposed a novel approach, Backwards Constraint Propagation (BCP), that automatically analyzes ETL workflows and verifies targetend integrity constraints at their earliest possible points.

1. BCP analyzes ETL workflows and target-end restrictions using an abstract interpretation, ETL graph.

2. It reports the earliest points in ETL workflows where these restrictions can be verified.

3. On detecting the violations of these restrictions, BCP calls predefined exception handlers.

4. BCP verifies whether ETL workflows contain contradictions.

Given an ETL process, $B C P$ builds an ETL graph, which extends the query tree in [3] and the system graph in [4, 5]. Then it pushes target-end restrictions backwards to the data sources by constraints projections [6, 7.

BCP can be used as a preprocessing step and applied to both categories of ETL tools. To evaluate its performance, we have implemented a prototype in our ETL tool OnceDI [8]. The preliminary results show that 1) BCP propagates most target-end restrictions back through ETL workflows, and places them at the earliest points where they can be verified; 2) our constraint propagation method supports all the relational algebra operators and data manipulation functions if they are monotonic and have inverse functions, which most involved functions in ETL workflows satisfy.

This paper is organized as follows. Section 2 gives a motivating example. Section 3 describes the BCP framework including constraints projection, automatic data quality rule generation and verification. Section 4 discusses related works, and Section 5 concludes the paper.

\section{One Motivating Example}

This section gives a motivating example, which is used as the running example in this paper.

Example 1. employee-department example: suppose a company has two headquarters, and both keep their employee and department information locally at two data sources $\mathrm{DS}_{1}$ and $\mathrm{DS}_{2}$. We want to integrate them into target data warehouse DW. 
$\mathrm{DS}_{1}$ is located in America, and contains two tables employee and department:

employee(EId, EName, Address, DeptId, StartDate, Salary) department(DeptId, DeptName, DirectorId, Revenue)

Relation employee has a tuple for every employee: EId is a unique integer ID assigned to each employee and the primary key; EName is the empoyee name; Address records the employee's home address; DeptId is the ID of the employee's department and a foreign key referring to the attribute DeptId of table department; StartDate is the date when the employee started working in department DeptId, and it is of DATE type and "MM-DD-YYYY" format; Salary is the annual salary in US dollars. Relation department has a tuple for every department: DeptId is a unique integer ID assigned to each department and the primary key; DeptName is department name; DirectorId is the ID of the department's director and a foreign key referring to the attribute EId of table employee; Revenue is the annual revenue of the department in US dollars.

$\mathrm{DS}_{2}$ is located in China, and also contains two tables employee and department:

employee(EId, EName, DeptId, StartDate, Salary) department (DeptId, DeptName, DirectorId, Revenue)

Their differences from $\mathrm{DS}_{1}$ are: 1) attribute Address is not recorded; 2) StartDate is of "YYYY-MM-DD" format, such as "2001-11-24"; 3) Salary and Revenue are in Chinese RMB instead of US dollars.

DW is located in America, and contains one relation defined as:

$$
\begin{array}{r}
\text { seniorEmpInLargeDept (EId, EName, StartDate, Salary, } \\
\text { DeptName, Size, Revenue, Source) }
\end{array}
$$

It has a tuple for each employee, and the primary key is EId. StartDate is in the format of MM-DD-YYYY, Salary and Revenue are in US dollars, Size is the number of employees in department DeptName, and Source denotes its data source.

In this problem data from both sources have to be processed before being loaded into DW. Without loss of generality, we used the ETL logic model introduced in [9] to represent the ETL process. The ETL workflow is shown in Figure 2. where triangles represent SQL operations or data manipulation functions. Assume there is an intermediate result relation resultTable(X) of an operation (triangle) $\mathrm{X}$. We detail each operation as:

A1: join tables employee and department from $\mathrm{DS}_{1}$ on DeptID.

A2: count (aggregate operation) EId as Size grouping by DeptId, DeptName, Revenue.

A3: join tables resultTable(A2) and employee from $\mathrm{DS}_{1}$.

A4: add attribute Source to table resultTable(A3), and set its values as " $\mathrm{DS}_{1}$ ".

A5: project out attribute DeptId from table resultTable(A4), get resultTable(A5) (EId, EName, StartDate, Salary, DeptName, Size, Revenue, Source). 
B1-B5: similar to A1-A5.

B6: convert StartDate format from "YYYY-MM-DD" to "MM-DD-YYYY".

B7: convert Salary from Chinese RMB to US dollars.

B8: convert Revenue from Chinese RMB to US dollars.

C1: union tables resultTable(A5) and resultTable(B8).

Suppose the set of restrictions at the target ic(DW) consists of the following five integrity constraints:

DW-IC1: all employees have a minimal salary of 50,000 US dollars.

DW-IC2: all departments have a minimal revenue of 1,000,000 US dollars.

DW-IC3: all departments have at least 10 employees.

DW-IC4: notNull(Salary), salary can not be NULL.

DW-IC5: pk(EId), EId is the primary key.

In order for the data arriving at the target warehouse to satisfy the set of integrity constraints ic $(\mathrm{DW})$, the data at a certain point $\mathrm{p}$ in the workflow should have some properties derivable from ic(DW). These properties can be encoded as a set of integrity constraints $i c(p)$ and verified at point $p$. Our approach to derive the integrity constraints over an ETL workflow and to place them at appropriate points is based on its ETL graph (an abstract interpretation).

An ETL graph is a labeled directed acyclic graph (DAG). The construction algorithm is formalized in Section 3. There are two types of nodes: relation nodes and axiom nodes. Relation nodes represent relations, and axiom nodes represent SQL operations or data manipulation functions. There are a set of data quality rules rules $(\alpha)$ and exception handlers handlers $(\alpha)$ associated with each relation node $\alpha$, and a set of mapping rules with each axiom node. The mapping rules of an axiom node $\beta$, mappings $(\beta)$, is an abstract interpretation of the operation $\beta$. Edges capture the interactions between relation nodes and axiom nodes. Edges are also referred as ports: incoming edges are referred as input ports and outgoing edges as output ports. The intuition is that data goes from data sources to the target along the edges. Relation nodes receive tuples from input ports, verify its set of data quality rules, and send tuples to output ports

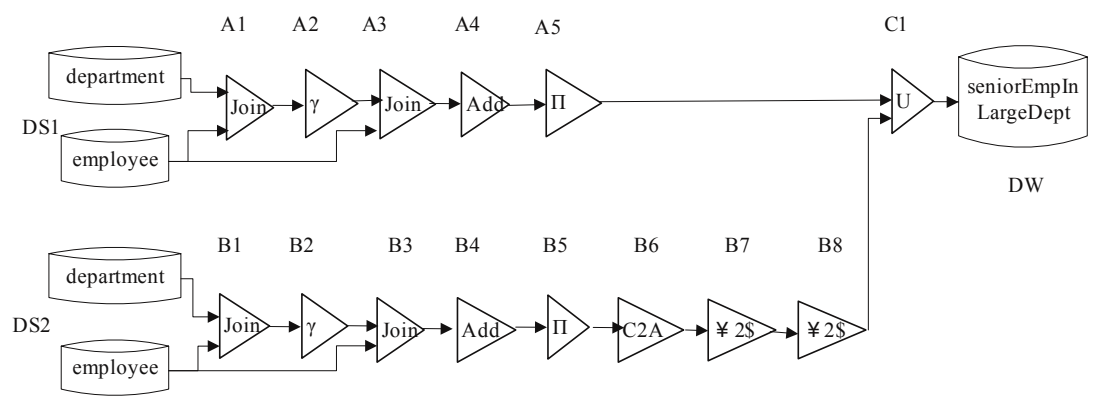

Fig. 2. ETL Workflow of Example 1 


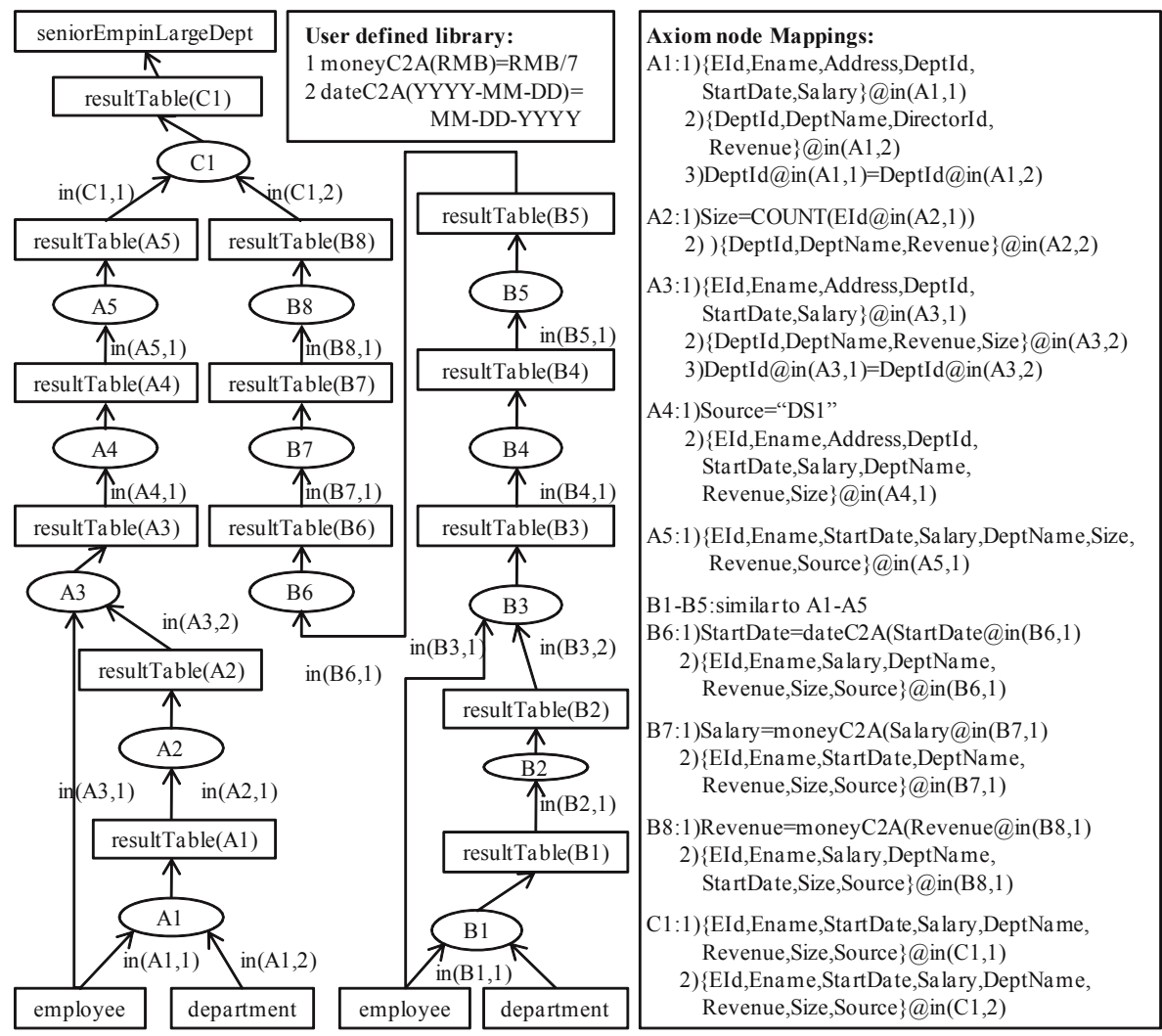

Fig. 3. ETL Graph of Example 1

if there is no exception. Otherwise, predefined exception handlers are called. Axiom nodes receive tuples from input ports, perform the stored operations, and send the resulting tuples to output ports.

The proposed ETL graph of the ETL process is shown in Figure 3. It also includes a user defined data transformation library, which defines how to perform the StartDate format conversion from China to America (function dateC2A), and how to convert Chinese RMB to US dollars (function moneyC2A).

BCP builds an ETL graph out of given ETL workflow, automatically derives the data quality rules $\operatorname{rules}(\alpha)$ of relation node $\alpha$, and pushes rules $(\alpha)$ backwards to data sources against the directions of data flows by applying constraint projection rules. Our constraint projection rules can push the constraints further back to data sources through functions if they are bijective and monotonic. Consider DW-IC1, at axiom node B7, we know that

$$
\text { Salary = moneyC2A(Salary@in }(B 7,1)) \text {, }
$$

and function moneyC2A is bijective and monotonically increasing, which gives us

$$
\text { moneyC2A(Salary@in }(B 7,1)) \geq 50,000 \text {. }
$$


After applying simple mathematical calculation, we know the incoming tuples to axiom node $B 7$ from input port in $(B 7,1)$ should have the property that

$$
\text { Salary } \geq 350,000
$$

which therefore is pushed to resultTable (B6) by adding it to rules (resultTable (B6)). Finally we can push this constraint down to $\mathrm{DS}_{2}$, which is the earliest place it can be verified.

\section{ETL Workflow Analysis and Verification}

\subsection{BCP Framework}

A framewrok is presented in Figure 4 to demonstrate how to integrate BCP into current ETL tools. The input of BCP module is the ETL graph of and targetend integrity constraints. The output is the data quality rules of each relation node. One step constraint projection algorithm is applied to each atom node to push constraints backwards. The redundancy resolve unit removes the redundant rules of each relation node. The implementation details of BCP in our ETL tool OnceDI and the preliminary results can be found in [10, and they are omitted here due to space limitations.

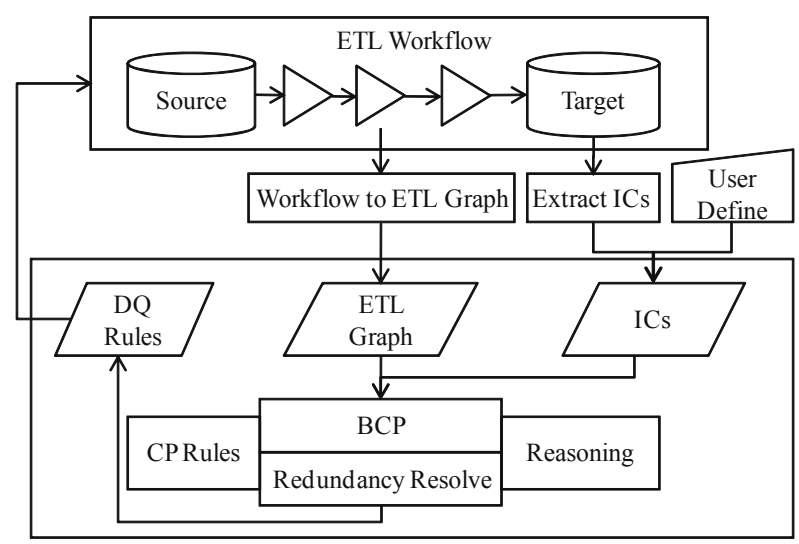

Fig. 4. BCP Framework

\subsection{ETL Graph}

ETL graph is an extension of the query tree in [3] and the system graph in [4, 5]. Before proceeding to the algorithm of ETL graph construction, we give some useful definitions (some have been used above informally).

Definition 1. Given an operation with label $\alpha$ in the given ETL workflow $\Omega$, its producers, producers $(\alpha)$, is the set of relations that provide data to operation $\alpha$, and its consumers, consumers $(\alpha)$, is the set of operations to whom operation $\alpha$ provides data. 
For instance, in Example 1 of Section 2] the producers of operation A3, producers $(\mathrm{A} 3)=\{$ resultTable $(\mathrm{A} 2)$, employee $\}$.

Definition 2. A data quality rule is an integrity constraint supported by DBMS (e.g., not null, unique, primary key, foreign key and check), or a user defined constraint of the form $R_{1}\left(\bar{X}_{1}\right) \wedge \ldots \wedge R_{n}\left(\bar{X}_{n}\right) \wedge \varphi\left(x_{1}, \ldots, x_{m}\right)$, where $R_{i}$ is a table, $\bar{X}_{i}(1 \leq i \leq n)$ is a vector of attributes, $x_{j}(1 \leq j \leq m)$ is a constant or an attribute in $\cup_{1 \leq i \leq n}\left(\bar{X}_{i}\right)$, and $\varphi$ is a built-in binary predicate that evaluates to true or false (e.g., $=, \leq, \geq$ ).

When the involved relations are obvious, we omit them when expressing a data quality rule. For example we used Salary $\geq 50,000$ without mentioning its involved relation seniorEmpInLargeDept in Example 1 of Section 2. The set of integrity constraints $\mathrm{ic}(\mathrm{DW})$ in Example 1 are encoded as data quality rules rules(DW): Salary $\geq 50,000, \quad$ Revenue $\geq 1,000,000, \quad$ Size $\geq 10$, notNull(Salary), and pk(EId).

Given an ETL workflow $\Omega$, the ETL graph $\mathrm{G}^{\Omega}$ is built in the following steps: 1 ) create an axiom node $\alpha$ for each SQL operation or data manipulation operation (triangle) $\alpha$ in $\Omega$, and compute mappings $(\alpha) ; 2$ ) create a result relation node resultTable $(\alpha)$ for each axiom node $\alpha$, initialize rules $(\operatorname{resultTable}(\alpha))=\emptyset$, and set its exception handling policies handlers(resultTable $(\alpha)) ; 3$ ) add edges between axiom nodes and relation nodes. The algorithm is as follows:

\section{Algorithm 1. ETL Graph Construction}

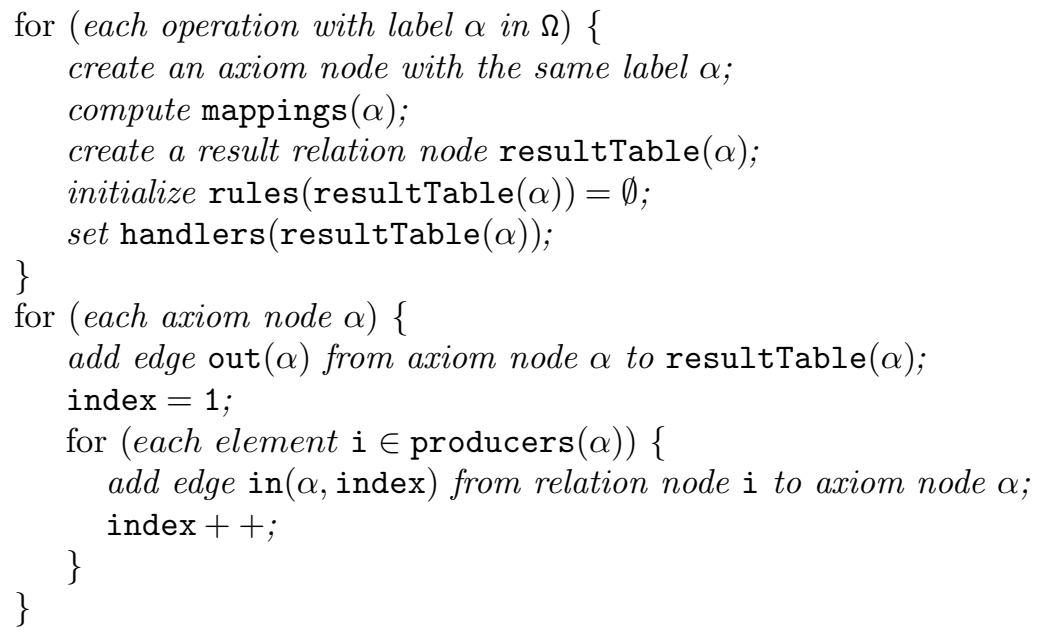

For a given atom node $\alpha$ associated with operation op, we suffix each table and its attributes in op with its input port label, and create mappings $(\alpha)$ to capture the mappings from its input attributes to output attributes. The mappings for different operations are generated differently as shown in Figure 5]. where $\operatorname{attrs}(\mathrm{X})$ returns the set of attributes involved in $\mathrm{X}$, which can be a relation, condition, rule, or function; annotate ( $\mathrm{X}$, inport) annotates each attribute y in X with its input port label by replacing y with y@inport; $\mathrm{S} @ i n(\alpha, \mathrm{i})$ 


\begin{tabular}{|c|c|c|}
\hline \multicolumn{2}{|r|}{ Operations } & Mappings \\
\hline Selection & $\begin{array}{l}\text { Select* } \\
\text { From rel@in } \\
\text { Where condition }\end{array}$ & $\begin{array}{l}\text { 1) attrs(rel)@in } \\
\text { 2) annotate(condition,in) }\end{array}$ \\
\hline Projection & $\begin{array}{l}\text { Select col1,...,coln } \\
\text { From rel@in }\end{array}$ & 1) $(\operatorname{col}, \ldots, \operatorname{coln}) @$ in \\
\hline Rename & $\begin{array}{l}\text { Select oldname as newname } \\
\text { From rel@in }\end{array}$ & $\begin{array}{l}\text { 1) newname=oldname@in } \\
\text { 2) attr(rel)-\{oldname }\} @ \text { in }\end{array}$ \\
\hline Add field & Add newname torel@in & 1) attrs(rel)@in \\
\hline Ordering & $\begin{array}{l}\text { Select * } \\
\text { From rel@in Order by .... }\end{array}$ & 1) attrs(rel)@in \\
\hline Aggregate & $\begin{array}{l}\text { Select groupByAttrs,f(oldname) as newname } \\
\text { From rel@in } \\
\text { Where condition } \\
\text { Group by groupByAttrs Having ... }\end{array}$ & $\begin{array}{l}\text { 1) } \quad \text { groupByAttrs }\} @ \text { in } \\
\text { 2) } \\
\text { 3) }\end{array}$ \\
\hline Function & newname $=\mathrm{f}($ oldname $)$ & $\begin{array}{l}\text { 1) newname }=\mathrm{f}(\text { oldname }) \\
\text { 2) } \operatorname{attrs}(\text { rel })-\{\text { oldname }\} @ \text { in }\end{array}$ \\
\hline $\begin{array}{l}\text { Join/ Intersection/ } \\
\text { Union/-/ } \times\end{array}$ & $\begin{array}{l}\text { rel1@in1 join/intersect/union } /-/ \times \\
\text { rel2@in2 }\end{array}$ & $\begin{array}{l}\text { 1) } \text { attrs(rel1)@in1 } \\
\text { 2) } \text { attrs(rel2)@in2 }\end{array}$ \\
\hline
\end{tabular}

Fig. 5. Mapping Generation of ETL Operations

means the set of output attributes $\mathrm{S}$ of axiom node $\alpha$ are from its input port in $(\alpha, i)$. For example of the second mapping of axiom node A3 in Figure 3 , $\{$ DeptId, DeptName, Revenue, Size $@$ in $(A 3,2)$ means that attributes DeptId, DeptName, Revenue, and Size are from input port in $(A 3,2)$.

\subsection{Constraint Propagation}

After the ETL graph of a given workflow is constructed, we initialize the set of data quality rules at the target warehouse (rules(DW) in Example 1) as its restrictions. Then our approach applies constraint projection rules to propagate these rules backwards to data sources. The constraint propagation algorithm support all relational algebra operators and most ETL workflow data manipulation functions (they are monotonic and have inverse functions).

Definition 3. The closure of a set of rules $\mathrm{S}$, closure(S), is the set of all rules that are logically implied by $\mathrm{S}$.

For example of rules $\mathrm{S}=\{\mathrm{x} \leq 10, \mathrm{x}=\mathrm{y}\}, \operatorname{closure}(\mathrm{S})=\{\mathrm{x} \leq 10, \mathrm{x}=\mathrm{y}, \mathrm{y} \leq 10\}$, since $\mathrm{y} \leq 10$ is logically implied by $\mathrm{S}$.

Definition 4. Given an axiom node $\alpha$ with input ports in $(\alpha, 1), \ldots$, in $(\alpha, \mathrm{n})$ from tables $\mathrm{table}_{1}, \ldots, \mathrm{table}_{\mathrm{n}}$ respectively, one step constraint projection 
$\Gamma(\alpha)$ propagates data quality rules rules $($ resultTable $(\alpha))$ to tables table ${ }_{1}, \ldots$, table $\mathrm{n}_{\mathrm{n}}$ through axiom node $\alpha$.

One step constraint projection $\Gamma(\alpha)$ works in the following three steps:

1. compute the union $\Psi(\alpha)=\operatorname{rules}(\operatorname{resultTable}(\alpha)) \cup \operatorname{mappings}(\alpha)$;

2. compute the closure of the union closure $(\Psi(\alpha))$;

3. project each data quality rule in closure $(\Psi(\alpha))$ onto every producer in $\operatorname{producers}(\alpha)$.

In step 1, before performing union, each mapping in mappings $(\alpha)$ of the format $\left\{\right.$ attr $_{1}, \ldots$, att $\left.r_{n}\right\} @$ inport is replaced with a set of rules $\left\{\right.$ attr $_{1}=$ attr $r_{1} @$

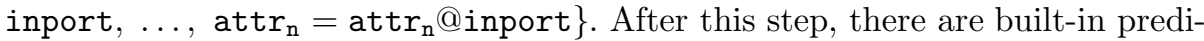
cates in $\Psi(\alpha)$. The attributes with "@inport" are from producers $(\alpha)$.

In step 2, we compute the closure closure $(\Psi(\alpha))$. If a contradiction is detected when computing the closure, the whole constraint propagation is aborted and an error is reported: there are errors in the ETL workflow design. For instance, if $\mathrm{x}<10 \in \mathrm{rules}(\mathrm{resultTable}(\alpha))$ and mappings $(\alpha)=\{\mathrm{x} @$ inport $>20$, $\mathrm{x}=\mathrm{x} @$ inport $\}$, then the union $\Psi(\alpha)=\{\mathrm{x}<10, \mathrm{x} @$ inport $>20, \mathrm{x}=\mathrm{x} @$ inport $\}$. We can infer that both $\mathrm{x} @$ inport $<10$ and $\mathrm{x} @$ inport $>20$ are in closure $(\Psi(\alpha))$, which is a contradiction.

Step 3 works differently for different data quality rules and axiom nodes. We elaborate below on how to project a data quality rule $r$ onto table table $e_{i}$ from input port in $(\alpha, i)$.

Operations without functions: selection, projection, rename, union, intersection, difference, product, join, not null, add field, ordering. There is no function application involved in these operations. Therefore, it is simpler to handle and the constraint projection rule, Rule 1, can be applied.

Rule 1. If $\operatorname{attrs}(r) \subseteq \operatorname{attrs}\left(\operatorname{table} e_{i}\right)$, then data quality rule $r$ is added to rules $\left(\mathrm{table}_{\mathrm{i}}\right)$.

Example 2. Consider the data quality rules in Example 1, we know:

$$
\begin{aligned}
\operatorname{rules}(\mathrm{DW})= & \operatorname{rules}(\operatorname{resultTable}(\mathrm{C} 1)) \\
= & \{\text { Salary } \geq 50,000, \text { Revenue } \geq 1,000,000, \\
& \text { Size } \geq 10, \operatorname{notNull}(\text { Salary }), \operatorname{pk}(\mathrm{EId})\} .
\end{aligned}
$$

Since operations $\{C 1, A 5, A 4\}$ do not change the involved set of attributes, by Rule 1, we get:

$$
\begin{aligned}
& \text { rules }(\operatorname{resultTable}(\mathrm{C} 1))=\operatorname{rules}(\operatorname{resultTable}(\mathrm{A} 5))= \\
& \text { rules }(\operatorname{resultTable}(\mathrm{A} 4))=\operatorname{rules}(\operatorname{resultTable}(\mathrm{A} 3)) .
\end{aligned}
$$

But operation A3 changes the involved set of attributes via mappings(A3), which pushes rules (resultTable (A3)) onto input port in $(A 3,2)$ as:

$$
\text { rules }(\operatorname{resultTable}(\mathrm{A} 2))=\{\text { Revenue } \geq 1,000,000, \text { Size } \geq 10\}
$$


We also notice that some rules are relaxed in this process. If a data quality rule involves more than one tuples in some relation, it is relaxed when it is pushed through union, intersection, product, and join. For example, pk(EId) in rules(resultTable(A5)) and pk(EId) in rules(resultTable(B8)) cannot guarantee $\mathrm{pk}(\mathrm{EId})$ in rules(DW).

Operations with functions: aggregation, function application. There are function applications involved in these operations, and thus more complex.

Rule 2.1. If axiom node $\alpha$ is aggregate operation on table $e_{i} \min ($ attr $)$ and $r$ is $\min ($ attr $) \geq$ val (or $\min ($ attr $)>$ val), then rule attr $\geq$ val (or attr $>$ val) is added to rules $\left(\right.$ table $_{\mathrm{i}}$ ).

Rule 2.2. If axiom node $\alpha$ is aggregate operation on table $e_{i} \max ($ attr $)$ and $r$ is $\max ($ attr $) \leq$ val $($ or $\max ($ attr $)<$ val $)$, then rule attr $\leq$ val (or attr $<$ val) is added to rules $\left(\right.$ table $\left._{i}\right)$.

Rule 2.3. If axiom node $\alpha$ is aggregate operation on table $e_{i}$ which is grouped by groupByAttrs and $\operatorname{attrs}(r) \subseteq$ goupeByAttrs, then data quality rule $r$ is added to rules $\left(\operatorname{table}_{i}\right)$.

Example 3. In Example 2, we know rules(resultTable(A2)) $=\{$ Revenue $\geq$ $1,000,000$, Size $\geq 10\}$. Axiom node A2 is an aggregateion operation on attributes $\{$ DeptId, DeptName, Revenue , by Rule 2.3, Revenue $\geq 1,000,000$ is pushed down to resultTable(A1), while Size $\geq 10$ is not.

Rule 3. If axiom node $\alpha$ is a function application newname $=f($ oldname $)$, $f$ has an inverse function and monotonic, and $\operatorname{attrs}(r) \subseteq \operatorname{attrs}\left(\operatorname{table} e_{i}\right)$, then replace $(r$, newname, $f(o l d n a m e))$ is added to rules $\left(t_{a b l e} e_{i}\right)$, where replace $(r$, newname, $f(o l d n a m e))$ replaces every occurrence of newname in rule $r$ with $f$ (oldname).

Example 4. In Example 1, we know that

$$
\begin{aligned}
\operatorname{rules}(\operatorname{resultTable}(\mathrm{B} 8))=\{ & \text { Salary } \geq 50,000, \text { Revenue } \geq 1,000,000, \\
& \text { Size } \geq 10, \operatorname{notNull}(\text { Salary }), \operatorname{pk}(\text { EId })\} .
\end{aligned}
$$

Axiom node B8 coverts Revenue from Chinese RMB to US dollars by applying

$$
\begin{aligned}
\text { Revenue } & =\text { moneyC2A }(\text { Revenue@in }(B 8,1)) \\
& =\text { Revenue@in }(B 8,1) / 7
\end{aligned}
$$

We know moneyC2A is monotonically increasing and bijective. By Rule 3, it can be pushed down to resultTable(B7) as

$$
\text { Revenue@in }(\mathrm{B} 8,1) \geq 7,000,000
$$

A similar process is applied to axiom node B7 for the conversion of attribute Salary, as shown in Example 1 of Section 2 . 
Let queue candidates contain the set of relation nodes whose rules are still to be pushed backwards. After defining one step constraint projection rules, we formalize the proposed backwards constraint propagation algorithm below.

\section{Algorithm 2. Constraint Propagation}

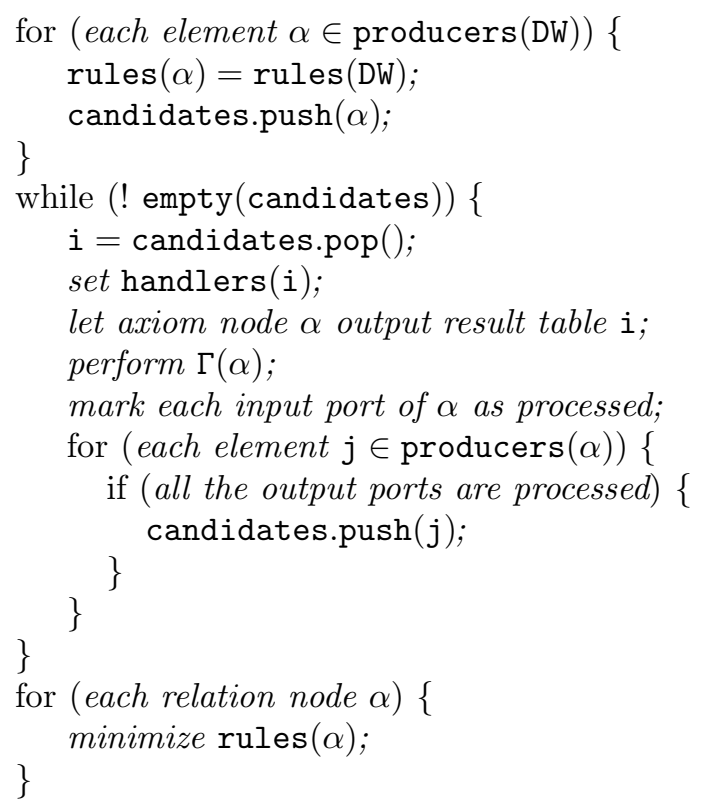

The last step of constraint propagation is to minimize the set of data quality rules at each relation node. There are two kinds of redundancies: 1) when computing closure, we may introduce more than necessary rules; 2) when rules(resultTable $(\alpha)$ ) are pushed down to producers $(\alpha)$, where they are verified, it is redundant to verify them again at resultTable $(\alpha)$. Redundancies of the first kind can be removed by the known technique transitive reduction, which repeatedly removes a rule if it is logically implied by the rest of the closure. For the second kind of redundancies, if a data quality rule $r$ in rules(resultTable $(\alpha)$ ) is projected onto all producers $(\alpha)$ and not relaxed, it is removed.

Example 5. Consider Example 1, after constraint propagation, we get handlers and rules of each relation node. The handler handler $(\alpha)$ of relation node $\alpha$ is to keep records of data exceptions. The rules of each relation node are:

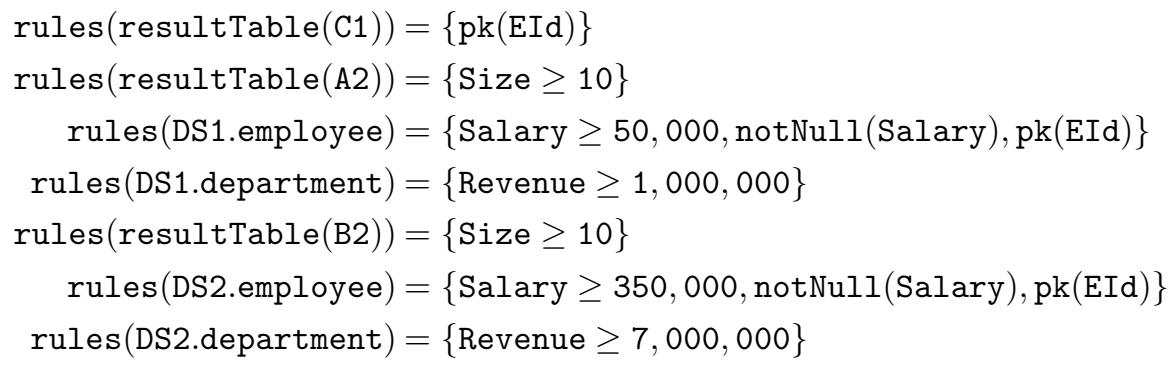


The rules of all other relation nodes are $\emptyset$. The data quality rules are propagated down to their earliest verifiable nodes.

\subsection{Correctness and Complexity}

Theorem 1. Suppose $\Gamma(\alpha)$ projects data quality rule $\mathbf{r}$ onto table table $\mathrm{e}_{\mathrm{i}}$ as $\boldsymbol{r}_{\mathrm{i}}$. If resultTable $(\alpha)$ satisfies $r$, then table $e_{i}$ satisfies $r_{i}$. If $\mathrm{table}_{\mathrm{i}}$ does not satisfy $r_{i}$, then resultTable $(\alpha)$ does not satisfy $r$.

Proof. The basic idea is that: for an axiom node $\alpha$, in order that resultTable $(\alpha)$ satisfies its rules, each table in producers $(\alpha)$ should provide data that satisfies both the projections of rules(resultTable $(\alpha))$ and the operation conditions, which are captured by mappings $(\alpha)$.

Theorem 2. One step constraint projection $\Gamma(\alpha)$ finishes in polynomial time of the number of rules.

Proof. Step 1 finishes in constant time. Step 2 finishes in polynomial time. Step 3 finishes in constant time. Overall $\Gamma(\alpha)$ finishes in polynomial time.

Theorem 3. Constraint propagation finish in polynomial time.

Proof. From Theorem 2, $\Gamma(\alpha)$ finishes in polynomial time, and constraint propagation performs one step constraint projection only once for each constraint. Overall constraint propagation finishes in polynomial time.

\section{Related Works}

There have been some studies in data cleaning and data quality control in ETL workflows, both industrial and academic. We discuss related works below.

ETL workflow design: The ETL market is increasing rapidly and so are the powerful features of leading data integration tools [11. SSIS from Microsoft supports data cleaning operations such as duplicate removal and exceptional data filtering. Oracle Data Integrator allows users to define filters at data sources. Some other ETL tools supports modular design and users can plug in their own data quality control module, such as Informatica Data Quality 5 allows business owners to configure data quality control processes. It supports data analysis, cleaning, matching and data monitoring. None of these system can perform ETL workflow analysis and target-end restriction verification automatically, which is the focus of this paper.

12 presented a generic and customizable framework of ETL workflow designs. They proposed a metamodel to define ETL activities, and employed declarative database programming language, LDL, to define ETL activity semantics. We used this framework in design the ETL workflow of Example 1.

\footnotetext{
${ }^{5}$ http://www.informatica.com/products_services/data_quality/Pages/index.aspx
} 
Query optimization: ETL workflow design can be optimized using predicate pushdown techniques [13]. 3] generalized this techniques to handle aggregations and other constructs such as NOT EXISTS. Their methods move predicates bottom-up and then top-down in the query tree, and thereby enables moving predicates to applicable nodes across the whole query tree. 5 ] further generalized this techniques to query deductive databases. Deductive database programs are modeled as system graphs, and the evaluation of queries are viewed as data flows in system graphs. They impose filters on edges to verify predicates. [9] modeled the logical optimization of ETL workflows as state-space search problems. Two unary activities can be swapped if it does not affect results. This paper focuses propagating target-end restrictions backwards to data sources. Although the resulting data quality rules help to optimize the design of ETL workflows, it is not our main topic.

Consistent Query Answering: It seeks consistent and correct answers when there are data exceptions. Some techniques perform query rewriting to incorporate the given integrity constraints. 14 gave a comprehensive survey. 15] studied data integration with integrity constraints. They focused on global schema analysis with primary key and foreign key constraints, and presented techniques to effectively answer queries in this situation. This paper is dealing with a more complete set of integrity constraints, not just primary and foreign key analysis.

\section{Conclusions}

In this paper, an approach (BCP) is proposed that automatically analyzes ETL workflows and verifies target-end restriction as early as possible. It supports all relation algebra operators and user defined functions if they are monotonic and bijective, which covers most ETL data transformation functions. Our analysis reports the set of integrity constraints that a given ETL workflow must satisfy at every point in the workflow, and it helps to optimize ETL workflow design. Future work includes extending the approach to support more complete ETL operations, implementing it completely in our ETL tool OnceDI, and run more extensive benchmarks.

Acknowledgements. This work was partially supported by the National Grand Fundamental Research 973 Program of China under Grant No.2009CB320704; the National Natural Science Foundation of China under Grant No.90718033, 60773028; National High-Tech Research and Development Plan of China under Grant No.2007AA01Z149 and No.2007AA04Z148. We also thank the anonymous reviewers for their very insightful comments.

\section{References}

1. Jarke, M., Lenzerini, M., Vassiliou, Y., Vassiliadis, P.: Fundamentals of Data Warehouses. Springer, New York (2001)

2. Rahm, E., Do, H.H.: Data cleaning: Problems and current approaches. IEEE Data Eng. Bull. 23(4), 3-13 (2000) 
3. Levy, A.Y., Mumick, I.S., Sagiv, Y.: Query optimization by predicate move-around. In: VLDB 1994: Proceedings of the 20th International Conference on Very Large Data Bases, pp. 96-107. Morgan Kaufmann, San Francisco (1994)

4. Kifer, M., Lozinskii, E.L.: Filtering data flow in deductive databases. In: Atzeni, P., Ausiello, G. (eds.) ICDT 1986. LNCS, vol. 243, pp. 186-202. Springer, Heidelberg (1986)

5. Kifer, M., Lozinskii, E.L.: On compile-time query optimization in deductive databases by means of static filtering. ACM Trans. Database Syst. 15(3), 385426 (1990)

6. Srivastava, D., Ramakrishnan, R.: Pushing constraint selections. In: PODS 1992: Proceedings of the eleventh ACM SIGACT-SIGMOD-SIGART symposium on Principles of database systems, pp. 301-315. ACM Press, New York (1992)

7. Marriott, K.G., Stuckey, P.J.: The 3 r's of optimizing constraint logic programs: refinement, removal and reordering. In: POPL 1993: Proceedings of the 20th ACM SIGPLAN-SIGACT symposium on Principles of programming languages, pp. 334344. ACM Press, New York (1993)

8. Jiangang, M., Dan, Y.: Data integration middleware. oncedi. 2.0. Technical Report (2004)

9. Simitsis, A., Vassiliadis, P., Sellis, T.: State-space optimization of etl workflows. IEEE Trans. on Knowl. and Data Eng. 17(10), 1404-1419 (2005)

10. Liu, J., Liang, S., Ye, D., Wei, J., Huang, T.: Etl workflow analysis and verification using backwards constraint propagation. Technical Report (2009)

11. Friedman, T., Beyer, M.A., Bitterer, A.: Magic quadrant for data integration tools (2008)

12. Vassiliadis, P., Simitsis, A., Georgantas, P., Terrovitis, M.: A framework for the design of etl scenarios. In: Eder, J., Missikoff, M. (eds.) CAiSE 2003. LNCS, vol. 2681, pp. 520-535. Springer, Heidelberg (2003)

13. Ullman, J.D.: Principles of Database and Knowledge-Base Systems, Volume 1 and 2. Computer Science Press, New York (1989)

14. Chomicki, J.: Consistent query answering: Five easy pieces. In: Proceedings of International Conference on Database Theory, pp. 68-76. Springer, Heidelberg (2007)

15. Calì, A., Calvanese, D., De Giacomo, G., Lenzerini, M.: Data integration under integrity constraints. In: Pidduck, A.B., Mylopoulos, J., Woo, C.C., Ozsu, M.T. (eds.) CAiSE 2002. LNCS, vol. 2348, pp. 262-279. Springer, Heidelberg (2002) 